\title{
Avaliação de Aditivos Poliméricos à Base de Éster Fosfórico como Modificadores da Cristalização de Parafina
}

\author{
Denise 0. Gentili \\ IMA, UFRJ / Centro de Pesquisas da Petrobrás, RJ \\ Carlos N. Khalil \\ Centro de Pesquisas da Petrobrás, $R \boldsymbol{R J}$ \\ Elizabete F. Lucas \\ IMA, UFRJ
}

\begin{abstract}
Resumo: O desafio da produção de óleos parafínicos e óleos pesados tem sido destaque no cenário de inovações tecnológicas na indústria do petróleo. Este trabalho apresenta a obtenção de um novo aditivo químico de base polimérica, e sua avaliação como modificador da cristalização de parafinas de petróleo. O polímero foi obtido pela reação de éster fosfórico de cadeia longa e aluminato de sódio, de modo a gerar uma molécula de peso molecular relativamente alto e de características anfifílicas. Os estudos foram realizados utilizando um sistema-modelo de parafina de petróleo (P140) dissolvida em solvente parafínico. Ensaios reológicos, calorimétricos, cromatográficos e de microscopia óptica e eletrônica de varredura evidenciaram a ação do aditivo como modificador da cristalização de parafinas, sendo que a eficiência se mostrou dependente do peso molecular do polímero.
\end{abstract}

Palavras-chave: Aditivos poliméricos, éster fosfórico, parafinas de petróleo, calorimetria, morfologia.

\section{Evaluation of Polymeric Phosphoric Ester-Based Additives as Modifiers of Paraffin Crystallization}

Abstract: The challenge of producing paraffinic and heavy oils plays an important role in the scenario of technological innovations in the petroleum industry. This work presents the synthesis of a new polymer-based chemical additive, and its evaluation as inhibitor of petroleum paraffin deposition. This polymer was obtained by reacting a long chain phosphoric ester with sodium aluminate, generating a molecule with relatively high molecular weight and amphiphilic character. The studies were carried out using a model-system of petroleum paraffin (P-140) dissolved in paraffin solvent. Rheological, calorimetric, chromatographic and optical and electron microscopy tests demonstrated that the additive acts as modifier of petroleum paraffin. The efficiency depended on the polymer molecular weight.

Keywords: Polymeric additives, phosphoric ester, petroleum paraffin, calorimetry, morphology.

\section{Introdução}

As companhias de petróleo têm intensificado a exploração offshore, onde os campos são localizados abaixo do fundo do mar. Neste caso, a baixa temperatura da água provoca um resfriamento brusco do óleo, ocasionando deposição de parafinas. $\mathrm{O}$ fenômeno de cristalização de parafina pode ser dividido em três estágios: a nucleação, onde o primeiro núcleo aparece; o segundo estágio, onde a massa produzida sai de solução; e o terceiro, onde ocorre a agregação dos cristais produzidos dando origem a cristais maiores ${ }^{[1-3]}$.

$\mathrm{O}$ fenômeno de precipitação de parafina pode ocorrer devido à ação de três mecanismos: (i) efeito termodinâmico, onde a redução de temperatura e o abaixamento da pressão provocam a precipitação e uma posterior deposição dos cris- tais que saem de solução; (ii) efeito da estrutura molecular, onde a linearidade da parafina e o seu alto peso molecular facilitam a sua agregação; (iii) efeito fluido-dinâmico, onde um regime turbulento, que provoca uma difusão molecular e uma dispersão cisalhante, favorece uma maior troca térmica e, conseqüentemente, a saída da parafina de solução; e um regime laminar, que provoca o ancoramento e aderência na parede e, ainda, alinha esses cristais favorecendo a deposição da parafina ${ }^{[4]}$.

Atualmente esse problema de deposição de parafina é controlado pela Petrobrás através de três métodos: (1) o preditivo, onde são feitas modelações moleculares e simulações numérica e física; (2) o preventivo, onde se emprega inibição química, inibição magnética e isolamento térmico; (3) o corretivo, onde são aplicadas uma remoção

Autor para correspondência: Elizabete F. Lucas, Instituto de Macromoléculas Professora Eloisa Mano, UFRJ, Caixa Postal 68525, CEP: 21945-970, Rio de Janeiro, RJ, Brasil.E-mail: elucas@ima.ufrj.br 
físico-química através de um sistema gerador de nitrogênio $(S G N)$ ou uma remoção mecânica com o uso de pigs ${ }^{[1,4]}$.

Tendo em vista que os problemas decorrentes do processo de parafinação não são solucionados de forma única, e estão fortemente associados às condições de escoamento e à natureza química do petróleo, diversas pesquisas científicas têm sido realizadas. Com o objetivo de otimizar o escoamento do petróleo, vários estudos comprovam que alguns aditivos poliméricos, especialmente copolímeros em bloco, têm a capacidade de alterar as propriedades reológicas do óleo. Esses aditivos inibem a deposição de parafina abaixo da temperatura inicial do aparecimento de cristais (TIAC) do óleo porque possuem, predominantemente, em sua estrutura uma porção alifática semelhante à da parafina, atraindo os cristais, e, em menor quantidade, um grupo diferenciador que impede a sua agregação. Uma grande vantagem na aplicação desses inibidores é a sua atuação com alto desempenho em concentrações extremamente baixas, sendo viáveis economicamente para o escoamento de petróleo em elevados volumes ${ }^{[1,2,4-7]}$.

Para óleos parafínicos, onde predominam os compostos saturados, os aditivos têm a capacidade de perturbar o crescimento dos cristais, alterando a sua morfologia de forma a impedir a deposição progressiva das parafinas. Conseqüentemente, a eficiência da adição de aditivos poliméricos pode ser monitorada pela não elevação da perda de carga por fricção durante o transporte do óleo. Assim, os cristais de parafina são formados em temperatura inferior a sua TIAC, porém estes não se depositam na parede interna do duto. Outro aspecto importante na aditivação química de petróleos parafínicos diz respeito à visível redução de viscosidade em temperaturas próximas a de ocorrência do fenômeno de parafinação. Para óleos pesados, onde predominam os aromáticos, asfaltenos e naftenos, acredita-se que os aditivos têm a capacidade de interagir com estes grupamentos, evitando a ligação entre os grupos polares de heteroátomos ou diminuindo a acidez naftênica do petróleo. $\mathrm{O}$ aditivo pode impedir o entrelaçamento dos dímeros, que podem ser formados devido à acidez naftênica, e que provocam altos valores de viscosidade. Em ambos os casos, os inibidores reduzem, significativamente, a viscosidade do petróleo ${ }^{[5,6,8]}$.

Este trabalho visa avaliar o desempenho de um aditivo químico capaz de promover eficientemente ações de modificação do hábito cristalino de parafinas de petróleo.

\section{Experimental}

\section{Reagentes}

Os reagentes para a obtenção do aditivo polimérico foram usados como recebidos: mistura de ésteres fosfóricos, a $43,7 \%$ p/v, e aluminato de sódio. Os dados de caracterização da mistura de ésteres e suas estruturas estão apresentados, respectivamente, na Tabela 1 e Figura $1^{[9]}$. Os constituintes do sistema-modelo, parafina P140 e solvente parafínico, foram obtidos da Refinaria de Duque de Caxias, Rio de Janeiro (Brasil) e usados como recebidos. A parafina P140 apresenta uma distribuição de $\mathrm{C}_{20}$ a $\mathrm{C}_{39}$ e o solvente parafínico de $\mathrm{C}_{10}$ a $\mathrm{C}_{17}$.
Tabela 1. Composição da mistura comercial de éster fosfórico ${ }^{[9]}$

\begin{tabular}{lccc}
\hline & \multicolumn{3}{c}{ Composição (\% em peso) } \\
\cline { 2 - 4 } & $\begin{array}{c}\text { Estrutura } \\
\text { I }\end{array}$ & $\begin{array}{c}\text { Estrutura } \\
\text { II }\end{array}$ & $\begin{array}{c}\text { Estrutura } \\
\text { III }\end{array}$ \\
\hline $\begin{array}{l}\text { Éster fosfórico } \\
\text { comercial }\end{array}$ & 1,4 & 23,3 & 19,0 \\
\hline
\end{tabular}

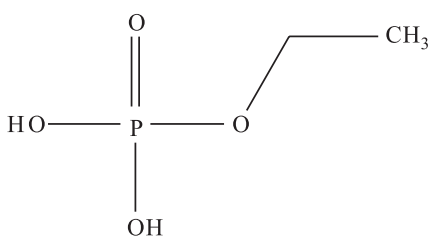

Estrutura I fosfato diácido de etila
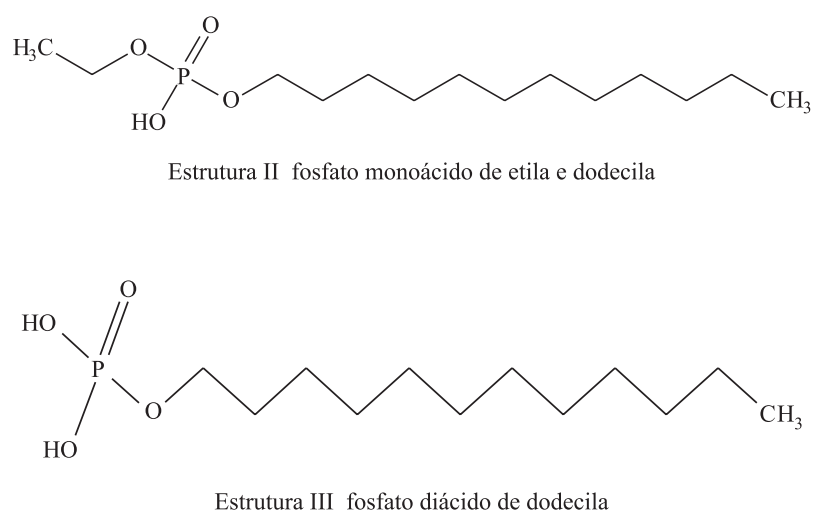

Figura 1. Estruturas químicas dos constituintes da mistura comercial de éster fosfórico

\section{Obtenção dos aditivos poliméricos}

Uma solução de éster fosfórico em querosene, a 1,3\%p/v, foi previamente preparada. Solução aquosa de aluminato de sódio (denominado ligante) foi adicionada a essa solução em diferentes quantidades. Foram preparados três aditivos poliméricos nas proporções (em massa) aluminato de sódio:éster fosfórico de $1: 10,5 ; 1: 8,7$ e 1:7,5. A reação foi realizada em sistema aberto, sob agitação magnética, a temperatura ambiente, por um período de 24 horas. A viscosidade do meio reacional foi monitorada utilizando um viscosímetro Brookfield LVDV-III.

\section{Preparo do sistema-modelo}

Foi preparada uma solução a 10\% de P140 em solvente parafínico: $10 \mathrm{~g}$ de P140 foram aquecidas até fusão e adicionadas a $90 \mathrm{~g}$ de solvente. A esta solução o aditivo polimérico foi, então, adicionado em concentrações de 50, 100, 150 e $200 \mathrm{ppm}$. Após a adição do aditivo, as soluções foram mantidas sob agitação por 15 minutos, sob aquecimento, para garantir a sua homogeneidade.

\section{Avaliação do desempenho do aditivo polimérico}

O sistema-modelo foi caracterizado quanto às propriedades reológicas em diversas temperaturas (de 40 a $10{ }^{\circ} \mathrm{C}$ ), 
empregando-se viscosímetro Brookfield LVDV-III, acoplado a um sistema de programação de aquecimento/ resfriamento.

Os valores de TIAC foram obtidos por calorimetria, utilizando um calorímetro modelo micro DSC III - Setaram, acoplado a um banho termostático, modelo phenix C25B. As amostras foram, primeiramente, aquecidas até $60^{\circ} \mathrm{C}$ e as curvas foram obtidas durante o resfriamento até $0{ }^{\circ} \mathrm{C}$, sob uma taxa de $1^{\circ} \mathrm{C} /$ minuto. $\mathrm{O}$ valor de TIAC foi tomado na interseção dos prolongamentos da linha base e do início de formação do pico, no resfriamento.

Os sistemas-modelo com e sem aditivo polimérico também foram avaliados por microscopia. Depois de homogeneizadas, sob aquecimento, as soluções foram transferidas para um capilar de vidro e vazadas na placa do microscópio. Em seguida, cada amostra foi submetida a aquecimento $\left(5^{\circ} \mathrm{C} / \mathrm{min}\right)$, para completa fusão dos cristais de parafina, e subseqüente resfriamento controlado $\left(0,5^{\circ} \mathrm{C} / \mathrm{min}\right)$. As análises de microscopia óptica $(\mathrm{OM})$ foram realizadas em um microscópio de luz polarizada, modelo Axioskop - Zeiss. As fotos foram registradas com uma lente de $20 \mathrm{X}$ de aumento.

As parafinas precipitadas em temperatura próxima a TIAC foram submetidas à análise morfológica, utilizando microscopia óptica, e análise de distribuição de parafinas por número de átomos de carbono, por meio de cromatografia gasosa com detector FID, (fabricado pela HP, modelo $5890 \mathrm{e}$ coluna capilar com fase estacionária de metil silicone e dimensões $30 \mathrm{~m} \times 0,25 \mathrm{~mm} \times 0,10 \mathrm{um})$. A separação das parafinas precipitadas dos sistemas-modelo com e sem a adição de aditivo polimérico foi realizada por meio de um processo de filtração, em três temperaturas distintas: 30,27 e $23{ }^{\circ} \mathrm{C}$ (todas correspondendo a um valor abaixo da TIAC).

\section{Resultados e Discussão}

\section{Obtenção dos aditivos poliméricos}

Os aditivos poliméricos foram obtidos pela reação da mistura comercial de ésteres fosfóricos e aluminato de sódio. As reações foram conduzidas com o objetivo de obter estruturas de peso molecular elevado contendo segmentos hidrocarbônicos (com afinidade pelas moléculas de parafina presentes do petróleo) e grupos polares (éster fosfórico) capazes de modificar o hábito cristalino dessas parafinas. A fim de serem obtidos produtos de pesos moleculares variados, para avaliar a influência do peso molecular no desempenho do produto como modificador da cristalização de parafinas, foram realizadas três reações com teores variados de reagentes. A Tabela 2 apresenta

Tabela 2. Nomenclatura dos produtos obtidos de acordo com as proporções de reagentes utilizadas

\begin{tabular}{cc}
\hline Produto & $\begin{array}{c}\text { Proporção em massa de aluminato } \\
\text { de sódio:éster fosfórico }\end{array}$ \\
\hline DG 1 & $1: 10,5$ \\
DG 2 & $1: 8,7$ \\
DG 3 & $1: 7,5$ \\
\hline
\end{tabular}

Tabela 3. Viscosidade dos sistemas reacionais em tempos variados

\begin{tabular}{cccc}
\hline \multirow{3}{*}{ Tempo (dias) } & \multicolumn{3}{c}{ Viscosidade (cP) } \\
\cline { 2 - 4 } & DG 1 & DG 2 & DG 3 \\
& $($ Al:P -1:10,5) & $($ Al:P - 1:8,7) & $($ Al:P - 1:7,5) \\
\hline 1 & - & 262,4 & 82,5 \\
2 & 296,2 & 488,1 & 299,2 \\
3 & 316,4 & 568,4 & 323,2 \\
4 & 335,9 & 619,4 & 378,7 \\
\hline
\end{tabular}

as proporções utilizadas em cada batelada e as respectivas denominações dadas a cada produto obtido.

Os sistemas reacionais tiveram suas viscosidades monitoradas ao longo de 4 dias (Tabela 3). Nos três sistemas, foi observado um aumento da viscosidade à medida que o tempo reacional aumentou, sugerindo um aumento de peso molecular. As reações foram realizadas com teores variados de éster fosfórico em relação ao aluminato do sódio com o objetivo de obter produtos de pesos moleculares também variados. Esperava-se que o peso molecular do produto aumentasse à medida que o teor de aluminato em relação ao éster aumentasse. Entretanto, pode-se observar para todos os tempos reacionais, que os maiores valores de viscosidade foram obtidos para o sistema polimérico do DG 2. Os resultados sugerem que existe um valor ótimo da razão aluminato de sódio/ éster fosfórico para obtenção de pesos moleculares mais elevados e esse valor está associado à relação estequiométrica desses reagentes que, neste caso, é de aproximadamente 1/10.

\section{Avaliação do sistema-modelo com e sem a adição de polímero}

A fim de estudar a influência dos aditivos sobre o comportamento reológico de sistemas contendo parafina e também sobre o ambiente dos cristais formados, optou-se por utilizar um sistema-modelo, constituído de parafina P140 dissolvida em n-parafina, a uma concentração de $10 \%$ p/v. A parafina P140 foi escolhida por representar as parafinas presentes no petróleo. O solvente n-parafina foi escolhido para simular a dissolução da P140 no petróleo. Este sistema-modelo permite a fácil visualização dos cristais formados, o que é muito difícil quando se utiliza um sistema real à base de óleo cru.

\section{Viscosidade}

O sistema-modelo teve seu comportamento reológico avaliado em teores de 50, 100, 150 e 200 ppm dos três tipos de aditivos obtidos. A Figura 2 apresenta as curvas de viscosidade em função da temperatura do sistema-modelo aditivado com DG 2. A viscosidade de todos os sistemas aumenta com a diminuição da temperatura. A utilização dos aditivos provoca uma queda nos valores de viscosidade medidos a baixas temperaturas. Os aditivos DG 2 e DG 3 foram mais eficientes na redução da viscosidade do que o aditivo DG 1 . $O$ aditivo DG 2 parece ser ligeiramente mais eficiente do que o DG 3. Além disso, observa-se um aumento de eficiência com o aumento 


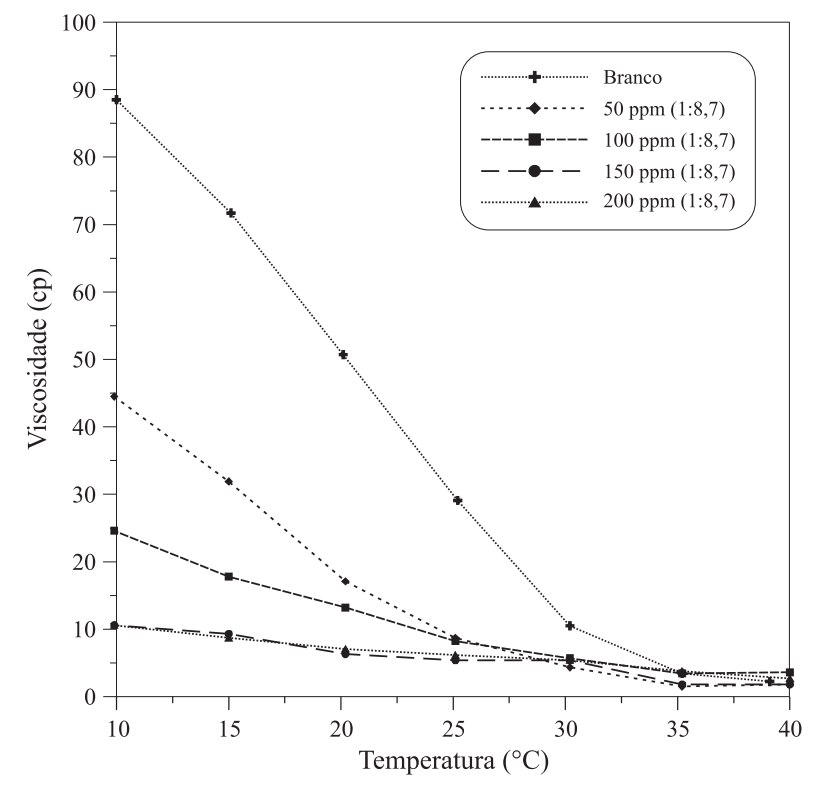

Figura 2. Curvas de viscosidade em função da temperatura do sistemamodelo com e sem a adição de DG2.

de concentração do aditivo, até o teor de 150 ppm. Esta concentração de aditivo pode ser a dosagem ótima, pois a 200 ppm não foi observada melhora na eficiência, mesmo porque, a $150 \mathrm{ppm}$, a viscosidade do sistema já permanece baixa com a variação de temperatura.

Como observado em outros trabalhos, os aditivos foram confeccionados para apresentarem segmentos hidrocarbônicos, capazes de interagir com as parafinas, e grupos polares, capazes de modificar seu hábito cristalino ${ }^{[2,6,10,11]}$. As amostras de aditivo, em princípio, diferem entre si somente quanto ao peso molecular, um parâmetro que também influencia na eficiência do aditivo ${ }^{[5,7,12,13]}$. Neste caso, parece que existe uma faixa de peso molecular ideal, uma vez que as amostras DG 2 e DG 3 (de pesos moleculares distintos) apresentaram desempenhos muito semelhantes, e a amostra DG 1, que possui peso molecular inferior, mas próximo ao peso molecular da amostra

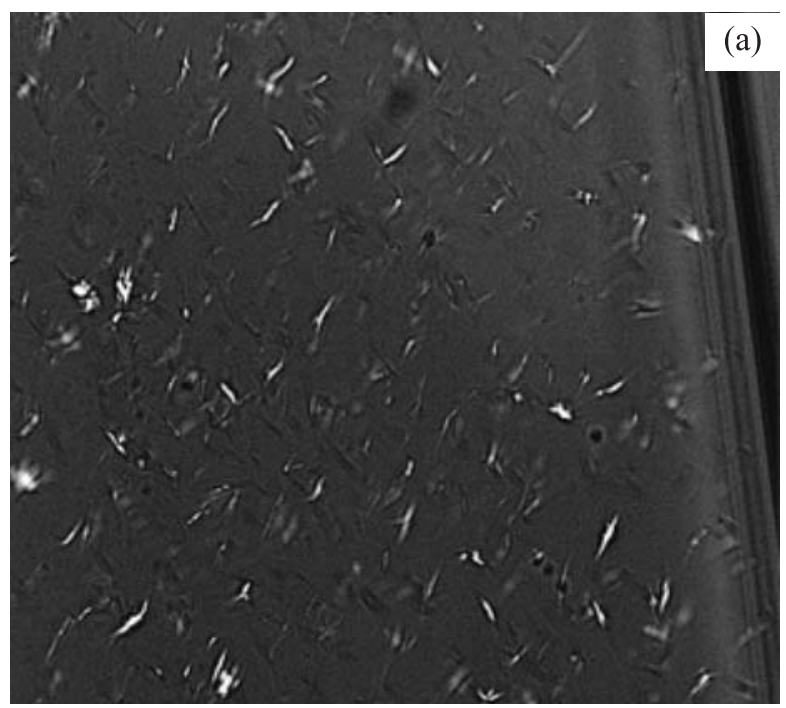

Tabela 4. Valores de TIAC e variação da entalpia de cristalização do sistemamodelo sem e com a adição de 200 ppm de aditivo

\begin{tabular}{ccc}
\hline Sistema-modelo aditivado & $\begin{array}{c}\text { TIAC } \\
\left(\mathbf{\pm 1}{ }^{\circ} \mathbf{C}\right)\end{array}$ & $\begin{array}{c}\Delta \mathbf{H}_{\mathbf{c}} \\
(\mathbf{1 ~ J} / \mathbf{g})\end{array}$ \\
\hline Puro & 34,3 & 16,9 \\
DG 1 & 34,9 & 16,2 \\
DG 2 & 34,9 & 16,6 \\
DG 3 & 35,0 & 16,9 \\
\hline
\end{tabular}

DG 3, apresentou o pior desempenho.

\section{Calorimetria diferencial de varredura (DSC)}

Os valores de TIAC dos sistemas e também o calor envolvido no processo de cristalização das parafinas foram obtidos por meio de medidas calorimétricas do sistema-modelo sem a adição de aditivo e com adição de cada um dos três aditivos, na concentração de 200 ppm (Tabela 4). Os resultados comprovam a formação de cristais em todos os sistemas, indicando que os aditivos não inibem a cristalização das parafinas. Os valores de TIAC não são alterados pela presença do aditivo, isto é, os cristais de parafina continuam a se formar na mesma temperatura, mesmo na presença do aditivo. Nenhuma variação é observada nos valores de entalpia de cristalização, o que não permite, por esta técnica, tirar conclusões a respeito da modificação do hábito cristalino induzida pela presença de aditivo.

\section{Microscopia ótica}

A microscopia óptica foi utilizada para verificar a morfologia dos cristais de parafina, na presença e na ausência de aditivo polimérico. Este estudo permitiu acompanhar o crescimento dos cristais observando seu tamanho e forma. As Figuras 3 e 4 mostram o comportamento da formação de cristais da parafina P140 com e sem a presença do aditivo, em duas temperaturas: próximo à TIAC $\left(35^{\circ} \mathrm{C}\right)$ e abaixo da TIAC $\left(25^{\circ} \mathrm{C}\right)$. A Figura 3 mostra o comportamento do siste-

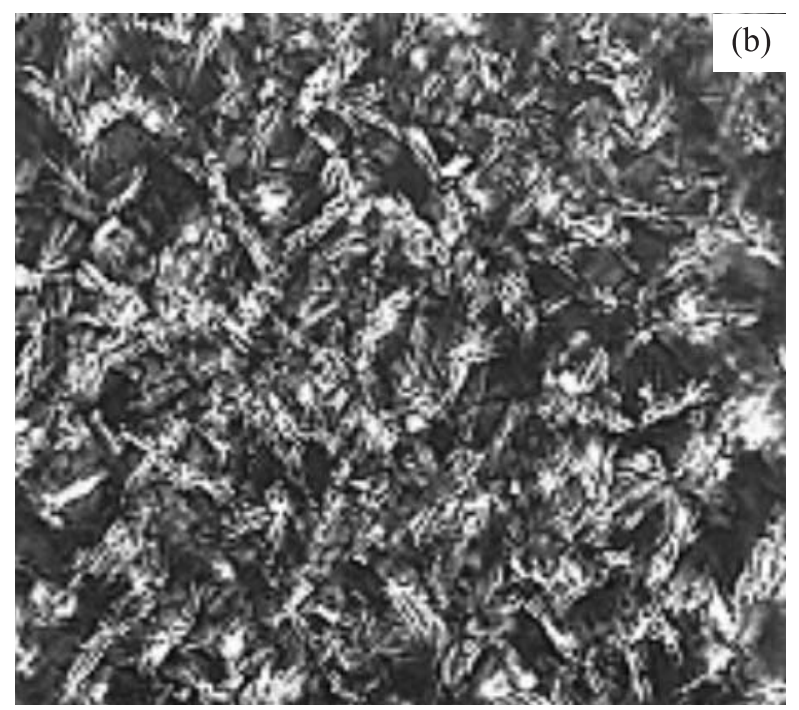

Figura 3. Microscopia óptica do sistema-modelo sem inibidor: (a) $35^{\circ} \mathrm{C}$ e (b) $25^{\circ} \mathrm{C}$. 

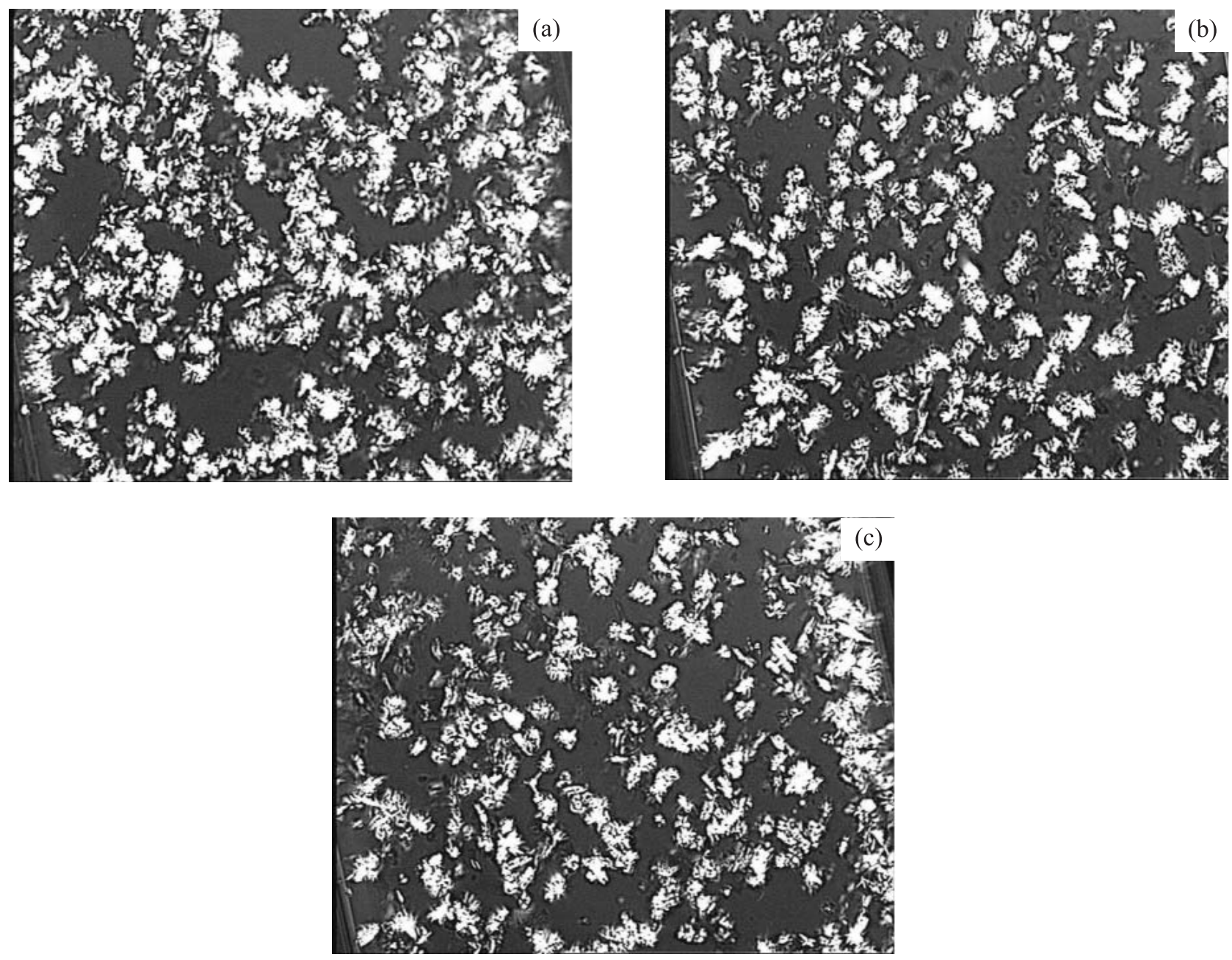

Figura 4. Microscopia óptica do sistema-modelo com (a) $200 \mathrm{ppm}$ de DG 1 a $25^{\circ} \mathrm{C}$, (b) $200 \mathrm{ppm}$ de DG 2 a $25^{\circ} \mathrm{C}$ e (c) 200 ppm de DG 3 a $25^{\circ} \mathrm{C}$.

ma modelo puro; na temperatura próxima à TIAC são observados alguns cristais bem pequenos e na temperatura abaixo da TIAC são observados muitos cristais na forma de agulhas, que são responsáveis pelos depósitos parafínicos que bloqueiam as linhas de produção de petróleo ${ }^{[1]}$. A morfologia desses cristais é modificada com a presença do aditivo; é observada a formação de cristais como se fossem pequenas "ilhas", que não se aglomeram para formar depósitos. A modificação do hábito cristalino é mais acentuada nos sistemas-modelo contendo os aditivos DG 2 e DG 3, o que está de acordo com os resultados de viscosidade que aumenta menos acentuadamente (com a redução da temperatura) na presença dos aditivos DG 2 e DG 3. Esses aditivos atuam na modificação do hábito cristalino evitando a aglomeração dos cristais para formar os depósitos. De acordo com os resultados de DSC, os cristais modificados são formados na mesma temperatura daqueles não-modificados e apresentam a mesma entalpia de fusão. Desse modo, acredita-se que as parafinas cristalizam do mesmo modo, e co-cristalizam com o aditivo que apenas modifica a superfície do cristal e não permite a aglomeração entre cristais de parafinas.

Comparando as micrografias do sistema-modelo aditivado com DG2 e DG 3, parece que o sistema contendo DG 2 apre- senta cristais de uma forma mais dispersa. Esta observação, associada aos resultados de viscosidade, indica que quanto maior for a capacidade do aditivo em dispersar os cristais de parafina, maior será a redução de viscosidade do sistema em temperaturas mais baixas, quando comparado ao ensaio em ausência do aditivo.

\section{Conclusões}

A reação de éster fosfórico com aluminato de sódio produz material de peso molecular elevado, sendo que um produto com peso molecular mais elevado é obtido a uma razão ótima de éster fosfórico e aluminato de sódio, a qual está próxima à relação estequiométrica dos reagentes. Os aditivos poliméricos obtidos da reação de éster fosfórico (contendo cadeias hidrocarbônicas longas) e aluminato de sódio agem com maior eficiência como modificadores da cristalização de parafinas se apresentarem peso molecular dentro de uma faixa ótima e forem utilizados também em concentração ótima, para o sistema-modelo adotado. Os aditivos poliméricos obtidos são capazes de manter a viscosidade, de um sistemamodelo de parafinas, relativamente baixa mesmo quando a temperatura é reduzida. A presença do aditivo polimérico 
modifica o processo de cristalização das parafinas e impede o fenômeno de aglomeração, responsável pela formação do depósito. A presença do aditivo polimérico parece não modificar o modo como as parafinas cristalizam, evitando somente a aglomeração dos cristais.

\section{Referências Bibliográficas}

1. Machado, A. L. C.; Lucas, E. F. - Petroleum Science and Technology, 19, p.197-204 (2001).

2. Price, R. C. - Journal of the Institute of Petroleum, 57, p.554 (1971).

3. Qian, J. W.; Qi, G. R.; Ding, X. Z.; Yang, S. L. - Fuel, 75, p.307-312 (1996).

4. Groffe, D.; Groffe, P.; Taknar, S.; Anderse, I.; Stenby, E. H.; Lindelof, N.; Lundgren, M. - Petroleum Science and Technology, 19, p.205-217 (2001).

5. Sunil Kumar, M.N.; Agrawal, Y.K. - Specialist Chemicals, 9, 321-326 (1989).

6. Khalil, C.N.; Gentili, D.O. - "Otimização do Escoamento de Óleos Movimentados pela Petrobras pela Utilização de Aditivos Poliméricos", Comunicação Técnica PDP/ TE-006/03, Centro de Pesquisas da Petrobras, Rio de Janeiro, Brasil (2003).

7. Donald, N.; Schulz, K.; Kitano, I.; Duvdevani, R. M.; Kowalik; Eckert, J. A. - "Hydrocarbon-Soluble
Associating Polymers as Antimisting and DragReducing agents", in: Polymers as Rheology Modifiers, Exxon Research and Engineering Company, cap.10, p.176-189, Annandle, N.J., EUA (1991).

8. Hamouda, A. A.; Strand, S. - "Flow Assurance for Transporting a Waxy Oil in an Integrated Complex Pipeline System and the Inherent Effect", in Rio Oil \& Gas Expo and Conference, Rio de Janeiro - RJ, out (2000).

9. Gentili, D. O. - "Avaliação de aditivos poliméricos à base de éster fosfórico como inibidores de deposição parafina", Tese de Mestrado, Universidade Federal do Rio de Janeiro, Brasil (2004).

10. Barthell, E.; Capelle, A.; Chmelir, M.; Dahmen, K. "Copolymers of N-Alkyl Acrylates and Maleic Anhydride and their Use as Crystallization Inhibitors for Paraffin-Bearing Crude Oils", Patente 4663491, EUA (1987).

11. Johnston, R. L.; Fry, L. G. - "Drag Reducers for Flowing Hydrocarbons”, Patente 5376697, EUA (1994).

12. Garcia, M. C.; Carbognani, L.; M. Orea; Urbina, A. - Journal of Petroleum Science \& Engineering; 25, p.99-105 (2000).

13. Figueredo, R. C. R.; Sabadini, E. - Colloids and Surfaces A: Physicochemical Engineering Aspects, 215, 77-86 (2002).

Enviado: 23/06/04 Aprovado: 30/08/04 